\title{
Comparison of the Laminar Distribution of Input from Areas 17 and 18 of the Visual Cortex to the Lateral Geniculate Nucleus of the Cat
}

\author{
P. C. Murphy, ${ }^{1,2}$ S. G. Duckett, ${ }^{1}$ and A. M. Sillito ${ }^{2}$ \\ ${ }^{1}$ Department of Physiology, St. George's Hospital Medical School, Tooting, London SW17 ORE, United Kingdom, and \\ 2Department of Visual Science, Institute of Ophthalmology, University College London, London EC1V 9EL, \\ United Kingdom
}

\begin{abstract}
The feedback from area 18 of the cat visual cortex to the lateral geniculate nucleus has been investigated by labeling and reconstructing seventeen axons of known receptive field position and eye preference. The distribution of boutons from each axon was quantified with respect to the compartments of the geniculate complex, and the results were compared with an equivalent analysis of fourteen area 17 axons. Area 18 axons form large, sparse arborizations that extend up to $1.9 \mathrm{~mm}$ laterally (1170 $\pm 85 \mu \mathrm{m}$; mean \pm SEM), with a core of relatively dense innervation spanning on average $600 \pm 70 \mu \mathrm{m}$ (mean \pm SEM). Thus, they have the potential to influence the transmission of visual information from well beyond their own classical receptive fields. In this respect, they are surprisingly similar to the axons from area 17, despite the fact that the two cortical areas have very different retinotopy. However, there are important
\end{abstract}

differences between the pathways. Area 18 axons project more heavily to the $\mathrm{C}$ layers and medial interlaminar nucleus. Whereas the input from both areas to the A layers is biased toward the layer appropriate to the eye preference of each axon, the area 18 input to magnocellular layer $C$ is not. The distribution of area 18 boutons favors the bottom of their preferred A layer, and the area 17 boutons favor the top. These differences mirror those seen in the afferent pathways, suggesting that each cortical area preferentially targets the cells from which it receives input. Finally, their greater diameter suggests that area 18 axons provide the earliest feedback signal in the corticogeniculate loop.

Key words: lateral geniculate nucleus; visual cortex; corticofugal feedback; functional connectivity; ocular dominance; visual responses
The lateral geniculate nucleus (dLGN) is the thalamic relay between the retina and the visual cortex, yet retinal afferents account for only a small minority of the synapses in the geniculate neuropil. Somewhat surprisingly, the greatest single source of input to the cat dLGN is the visual cortex itself (Wilson et al., 1984; Montero, 1991; Vidnyánszky and Hámori, 1994; Erisir et al., 1997, 1998). This corticofugal feedback pathway has been shown to exert a wide range of effects on the visual response properties of geniculate cells (Kalil and Chase, 1970; Richard et al., 1975; Schmeilau and Singer, 1977; Geisert et al., 1981; Murphy and Sillito, 1987, 1989; Varela and Singer, 1987), and there is a growing awareness that that it may play an important role in rephrasing subcortical processing in the context of the operations performed cortically (Marrocco et al., 1982; Sillito et al., 1993, 1994; Cudeiro and Sillito, 1996).

The interpretation of these results is complicated, however, by the fact that the feedback is heterogeneous. In the cat, it involves at least three separate cortical areas, 17, 18, and 19 (Kawamura et al., 1974; Updyke, 1975), each of which forms a characteristic pattern of corticofugal connections. In particular, although bulk labeling experiments have shown that the area 17 and 18 pathways both terminate in all of the layers and compartments of the

\footnotetext{
Received May 10, 1999; revised Oct. 4, 1999; accepted Oct. 29, 1999.

This work was supported by Medical Research Council Programme Grant G8519936. We thank Suzanne Claxton for her excellent technical assistance, and the British Physiological Society and St. George's Hospital Medical School for additional funding toward S.G.D.

Correspondence should be addressed to Penelope C. Murphy, Department of Physiology, St. George's Hospital Medical School, Cranmer Terrace, Tooting, London, SW17 0RE, UK. E-mail: p.murphy@sghms.ac.uk.

Copyright (C) 2000 Society for Neuroscience 0270-6474/00/200845-09\$15.00/0
}

dLGN complex, there is evidence to suggest subtle differences in their bouton distributions (Updyke, 1975) and choice of postsynaptic targets (Vidnyánszky and Hámori, 1994). Because areas 17 and 18 have different retinotopic organizations (Tusa et al., 1978, 1979; Cynader et al., 1987), afferent inputs (Höllander and Vanegas, 1977; LeVay and Ferster, 1977; Dreher et al., 1980; Geisert, 1980), visual response properties (Hubel and Wiesel, 1965; Tretter et al., 1975; Orban and Callens, 1977; Movshon et al., 1978; Orban and Kennedy, 1981; Orban et al., 1981a,b; Price et al., 1994), and response latencies (Tretter et al., 1975), it follows that each group of dLGN cells must receive a functionally distinct pattern of corticofugal influence.

To better understand the structural basis of the corticofugal signal, we set out to visualize, reconstruct, and quantify the individual elements of the feedback pathways from areas 17 and 18. We have shown previously that the axons arising from area 17 are heterogeneous but nevertheless share a characteristic pattern of connectivity within the laminar structure of the dLGN (Murphy and Sillito, 1996). In particular, the successfully stained axons were found to project primarily to the A layers and to show a marked bias toward the layer matching their eye preference. Thus, they target the regions from which they receive their own predominant input. The primary purpose of the experiments reported here was to provide analogous data for area 18 and to compare and contrast the organization of the two pathways at a single axon level. The results show that the pattern of connectivity for area 18 axons both resembles and differs from that of the area 17 population, in ways that have important implications for the functional organization of feedback pathways in general. 


\section{MATERIALS AND METHODS}

The experiments were performed on young ( $>14$ weeks old) adult cats, well beyond the age at which the corticof ugal pathway is thought to reach maturity (Weber and Kalil, 1987). The animals were anesthetized (70\% $\mathrm{N}_{2} \mathrm{O}, 30 \% \mathrm{O}_{2}$, and $0.1-0.4 \%$ halothane), paralyzed (gallamine triethiodide; $10 \mathrm{mg} \cdot \mathrm{kg}^{-1} \cdot \mathrm{hr}^{-1}$ ), and artificially ventilated so as to maintain end-tidal $\mathrm{CO}_{2}$ levels at between 3.8 and $4.2 \%$. Electrocardiogram waveform, intersystolic interval, and the frequency of EEG spindles were monitored continuously, and the halothane was adjusted to give a state of light anesthesia. Wound margins were treated with subcutaneous procaine hydrochloride, and the ear bars were coated with antiseptic lignocaine hydrochloride gel. The eyes were treated with atropine methonitrate and phenylephrine hydrochloride, protected with contact lenses, and focused on a semiopaque tangent screen at a distance of $1.14 \mathrm{~m}$. Additional details of our procedures are given elsewhere (Murphy et al., 1993).

Single corticofugal axons from layer VI of area 18 were labeled by extracellular injection of biocytin. The experiments were performed using micropipettes filled with a mixture of $2-4 \%$ biocytin (Sigma, St. Louis, MO) in Tris-buffered ( $\mathrm{pH} 7.2) \mathrm{KCl}$, tip size of $\sim 1 \mu \mathrm{m}$ diameter. Representative cells were recorded at each injection site, and their receptive fields were mapped. In this way, the injections were localized to the center of ocular dominance columns, so as to label axons with monocular, or nearly monocular, receptive fields (see also Murphy and Sillito, 1996). Cells were then stained by passing small currents $(+180-$ $275 \mathrm{nA}$ ) for long periods $(60-70 \mathrm{~min})$, using a $1 \mathrm{sec}$ on/1 sec off duty cycle.

After an 18-40 hr survival period, the animals were deeply anesthetized and perfused. Tissue blocks containing the stained cells and their processes were then prepared, frozen sectioned at $40 \mu \mathrm{m}$, and treated for the detection of biocytin as described previously (Murphy and Sillito, 1996). Cortical sections were counterstained with neutral red to establish laminar and areal borders. Geniculate sections were mounted, dehydrated, and coverslipped, and the stained axons were drawn and reconstructed using either a traditional camera lucida or a computer-linked three-dimensional analysis system running the Neurolucida software (MicroBrightField, Inc., Colchester, VT). After completion of the drawings, selected sections from within and around the axonal arborizations were also counterstained to confirm the location of the interlaminar zones. The injections typically stained a cluster of cells $<100 \mu \mathrm{m}$ wide, from which a smaller number of axons could be visualized as far as the dLGN. All of the labeled processes visible in the thalamic block were drawn and reconstructed. The great majority faded out soon after their first branch point. Of the remainder, only those axons that had well labeled intrageniculate collaterals that could be distinguished clearly from other processes arising from the same injection site were included in the detailed analysis. These were all from injections that had $>32 \mathrm{hr}$ survival time. The axons were not reconstructed back to their point of origin, so we cannot exclude the possibility that some might have branched in the segment of white matter bridging the cortical and thalamic tissue blocks. We think it unlikely, however, because we examined them over most of their course and never observed a branch point above the level of the thalamic reticular nucleus that produced two descending axons. The main incoming axons were examined close to the point at which they enter the thalamus, using a $100 \times$ oil immersion objective, and rank-ordered in terms of their perceived thickness. Their diameters were also estimated to within $0.25 \mu \mathrm{m}$. The accuracy of these measurements is inevitably limited by the resolution of the light microscope, but for the purposes of comparison between the two populations, the results are both robust and repeatable.

For each fully reconstructed axon, the total number and distribution of presumed synaptic boutons were determined with respect to the main layers and compartments of the dLGN complex, and the results were compared with those for a previously described population of area 17 axons. There is evidence that projections both to and from area 18 are unevenly distributed through the depth of the A layers (Updyke, 1975; Geisert, 1985; Erisir et al., 1998); therefore, for both the areas 17 and 18 axon populations, each A layer was subdivided into top, middle, and bottom thirds. To ensure that the mediolateral distribution of boutons could be assessed accurately, the layers were also divided into $90-\mu \mathrm{m}$ wide columns oriented perpendicular to the laminar borders. This produced a continuous grid, within which the total number of boutons from each axon within each compartment were counted separately. The number of compartments containing boutons provided a quantitative measure of the total extent of each arborization at each depth through the A

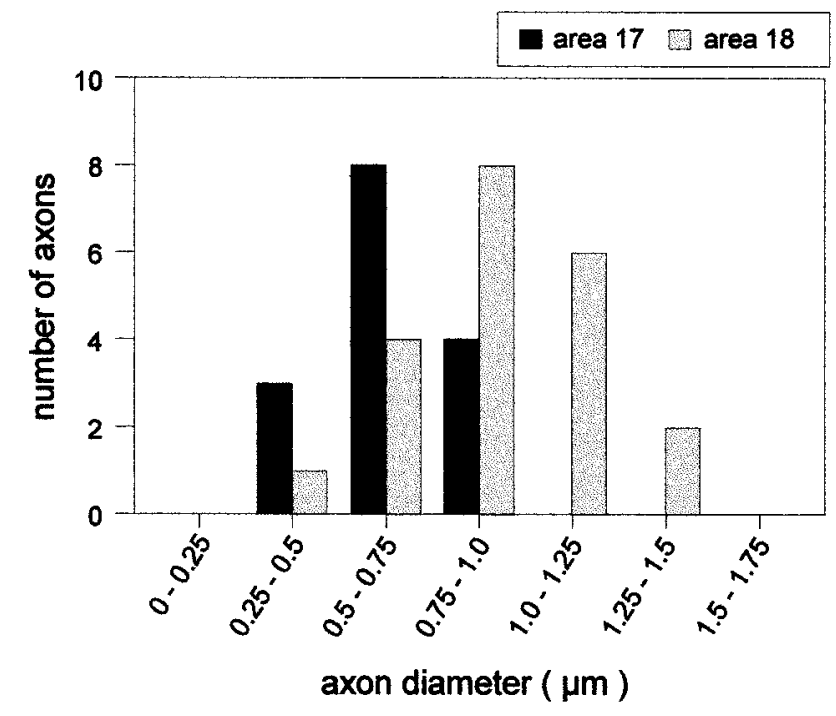

Figure 1. The distribution of axon diameters for area 17 and area 18 corticof ugal axons. Note that several incompletely stained, and therefore unreconstructed, axons have been included in this figure; hence, $n=15$ for area 17, and $n=21$ for area 18 . Area 18 axons are significantly thicker, and hence presumably faster, than those from area 17 (Student's $t$ test; $p<0.01)$.

layers; the number of boutons per compartment gave an indication of bouton distribution. Because it was impossible to apply an exactly equivalent method to both hand- and computer-drawn data, the sublayer analysis was confined to the hand-drawn axons only.

Finally, in a number of cases, carefully spaced penetrations were made into the dLGN with tungsten recording electrodes. The tracks were identified in the histological material, and their separation was used to estimate tissue shrinkage (histological measurements were $\sim 90 \%$ of the known in vivo values). All distances given in the illustrations and text have been corrected accordingly. The intrageniculate axons are extremely fine and delicate and have a very wide spread. To reduce the final reconstructions to a size suitable for publication, it was necessary to exaggerate the diameter of the branches and the size of their boutons and stalks. However, a more realistic depiction of individual collaterals can be found elsewhere (Guillery, 1966; Robson, 1983, 1984; Boyapati and Henry, 1984; McCart and Henry, 1994).

\section{RESULTS}

The data described below relate to seventeen area 18 corticof ugal axons, which were recovered from seven cats (one to five axons per cat). The cells of origin had receptive fields between 1.5 and $12^{\circ}$ of the area centralis, giving a range of eccentricities comparable with those of the fourteen area 17 axons described previously. The two populations have a broadly similar appearance at the light microscopic level. In either case, approximately half of the reconstructed axons split into two branches as they pass through the thalamic reticular nucleus, although both branches then project to the same region of the dLGN. The majority produce a few fine collaterals bearing boutons at this point. All of the axons innervate the region of the perigeniculate nucleus (PGN) immediately above, and hence retinotopically corresponding to, their dLGN projection zones. Thereafter, both populations form sparse but extensive arborizations of fine collaterals within the body of the dLGN, which have the typical type 1 morphology described by Guillery (1966).

One difference that is immediately obvious, however, is that the area 18 axons are thicker than those from area 17 (Fig. 1), while their intrageniculate collaterals appear to be stouter and more easily visualized. The difference in thickness, and hence presum- 

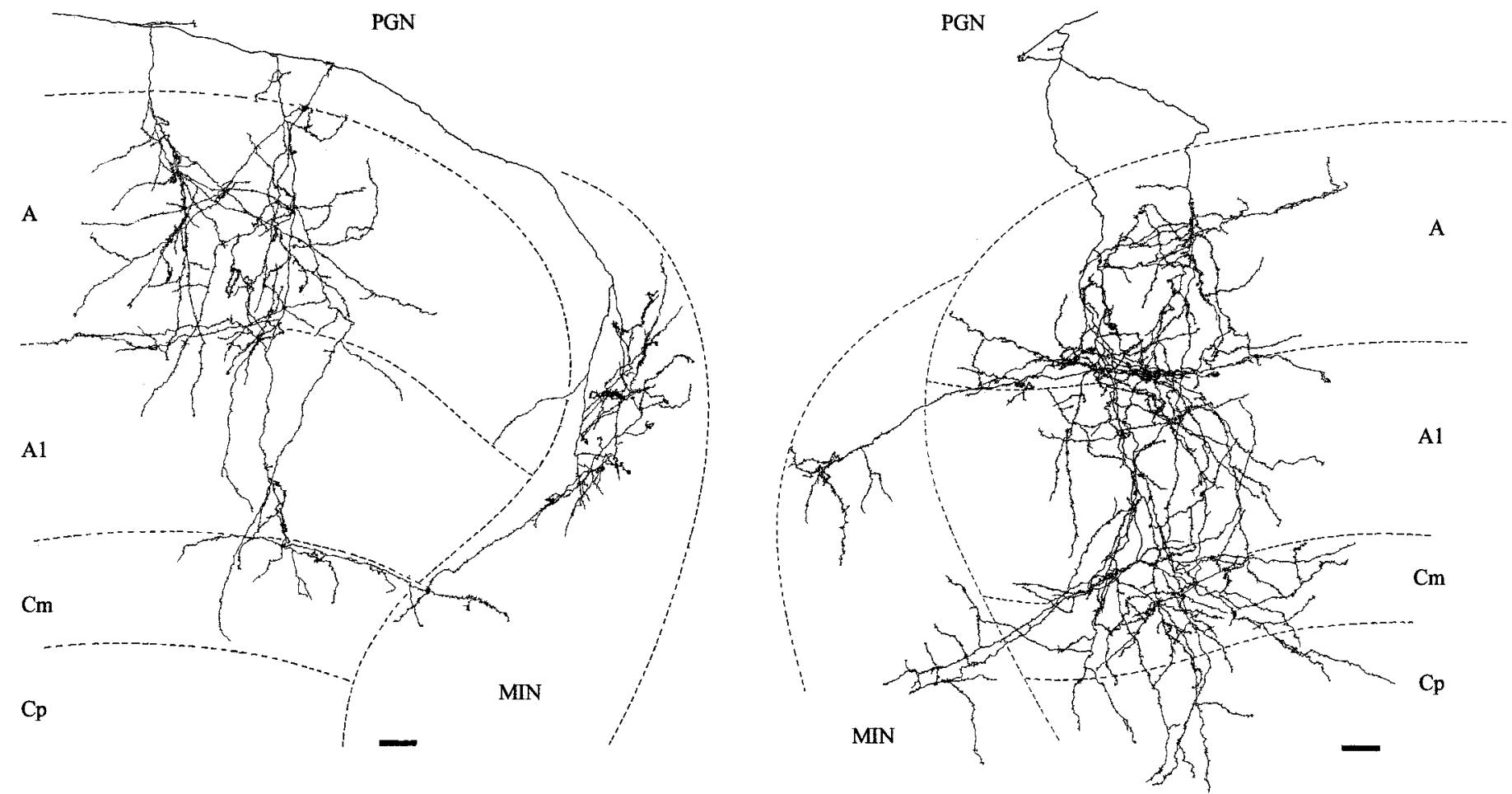

Figure 2. Reconstruction of two area 18 corticofugal axons. Both form widespread but sparse arborizations within layers A and A1, Cm, and MIN. One also projects well into the parvocellular $\mathrm{C}$ layers $(C p)$. This is the archetypal area 18 projection pattern. Both axons were stained by extracellular injections into the center of contralateral eye ocular dominance columns, and both show a bias in their projection in favor of the contralateral eye-dominated layer A compared with the ipsilateral eye-dominated layer A1. In the case of the second axon (right), this bias is small but is augmented by a strong projection into the contralateral eye-dominated layer $\mathrm{Cm}$. Note that, in both cases, the input to layer $\mathrm{A}$ is concentrated in the bottom two-thirds of the layer and that there are several long, horizontal branches in the vicinity of the laminar borders. For the sake of clarity in these drawings, it was necessary to exaggerate the diameter of even the thickest branches, the size of terminal swellings, and the length of their stalks. Scale bars, $100 \mu \mathrm{m}$.

ably in conduction velocity, is statistically significant (MannWhitney $U$ test; $p<0.01$ ). Beyond this, a full reconstruction and quantitative analysis of the axonal arborizations has revealed a number of important differences, as well as several points of surprising similarity, between the two pathways.

\section{Comparison of the pattern of input to different laminae}

The areas 17 and 18 axons differ most clearly with respect to the laminar distribution of their boutons, to the extent that the origin of any given axon can be recognized with ease. Archetypal examples of the area 18 projection pattern are shown in Figure 2. Like most of their kind (13 of 17), both axons give off a few fine collaterals as they pass through the thalamic reticular nucleus, and both provide some, albeit very sparse, innervation to the PGN. Within the dLGN itself, each axon forms a widespread network of collaterals that descends vertically through the geniculate layers. This description could apply equally well to axons from area 17. However, although our stained area 17 axons are primarily confined to the A layers, both of these axons form separate and distinct arborizations within the $\mathrm{C}$ layers and medial interlaminar nucleus (MIN). This clearly distinguishes them from any area 17 axon that we have stained.

Figures 3 and 4 show that this pattern is characteristic for the area 18 population as a whole and that the differences described above are both consistent and statistically significant. Figure 3 is a histogram illustrating the overall distribution of corticofugal boutons for each area 18 axon individually. The data are ranked according to the diameter of the parent axon, with the thickest to

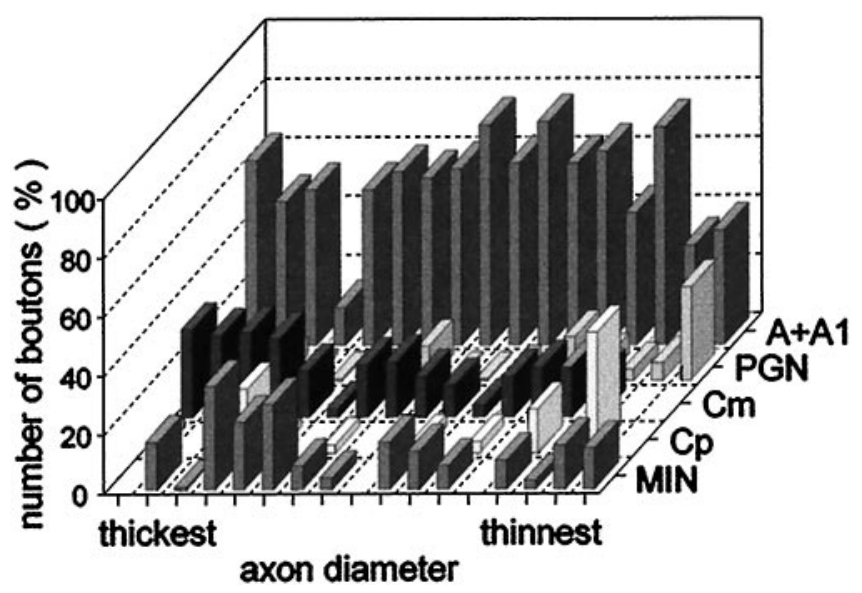

Figure 3. The distribution of boutons for each individual area 18 corticofugal axon, expressed as a percentage of the total for that axon, within the main layers and compartments of the dLGN complex. The data are ranked such that the thickest axon is to the left and the thinnest to the right. The $\mathrm{A}$ layers $(A+A 1)$ are the largest compartment and hence receive the greatest number of boutons, but each axon also makes a substantial contribution to the C layers and/or MIN. Indeed, bouton density frequently appears highest in $\mathrm{Cm}$ or MIN. $C p$, Parvocellular C layers.

the left and the thinnest to the right. Note that, although the majority of boutons are found in the A layers, this is primarily a reflection of the relative size of those layers. All of the axons provide a substantial input to other compartments, and in many 
area $17 \square$ area 18
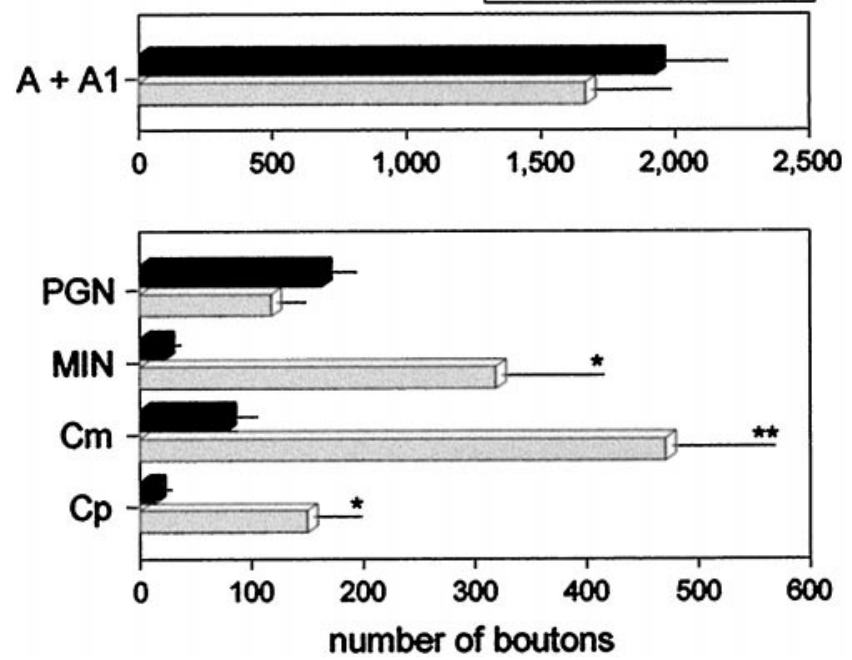

Figure 4. A comparison of the average number of boutons from areas 17 and 18 axons within the main layers and compartments of the dLGN complex. Error bars are 1 SEM; $n=14$ for area $17 ; n=17$ for area 18 . Note that area 18 provides a significantly greater innervation to the $\mathrm{C}$ layers and MIN (Student's $t$ test; * $p=0.01 ;{ }^{* *} p=0.001$ ). $C p$, Parvocellular C layers.

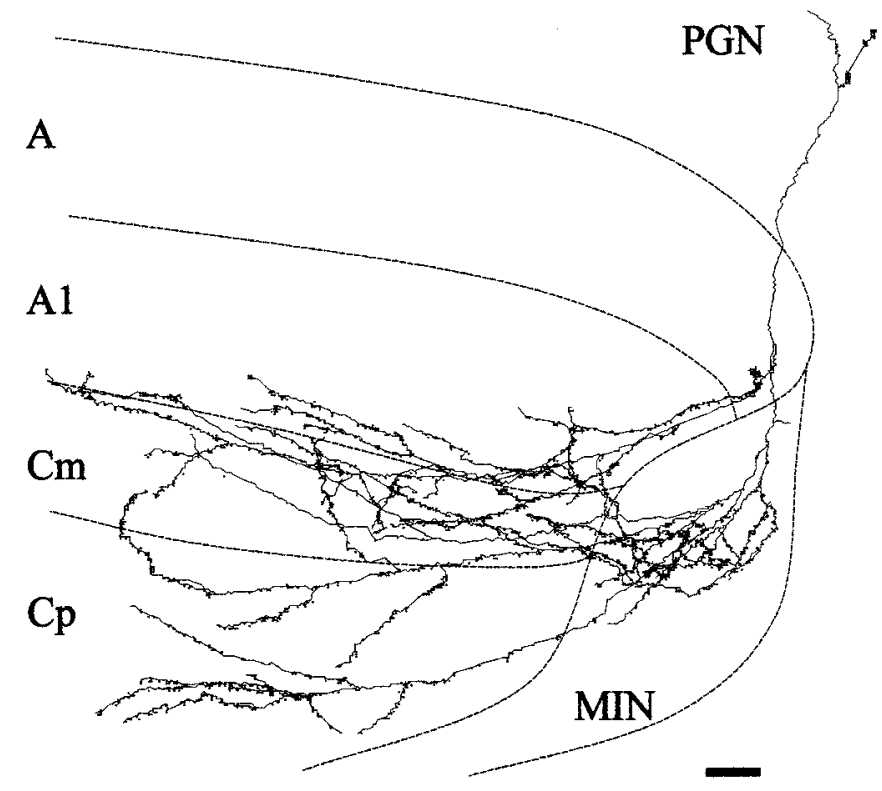

Figure 5. Reconstruction of a corticofugal axon that was stained by injection into the center of an ipsilateral eye ocular dominance column. This is an extreme example of the characteristic area 18 projection pattern, in that the axon primarily bypasses the A layers in favor of the $\mathrm{C}$ layers, both magnocellular and parvocellular, and MIN. Scale bar, 100 $\mu \mathrm{m}$. $C p$, Parvocellular C layers.

cases, bouton density actually appears highest in either magnocellular layer C (layer Cm) or MIN. Two axons in particular, one of which is illustrated in Figure 5, target the C layers and MIN in preference to the A layers. A few miss one or the other of these targets, but none miss both. For example, the axon illustrated in Figure 6 (left) does not extend into MIN, but the dense skirt of collaterals in layer $\mathrm{Cm}$ nevertheless distinguishes it as an area 18 axon. Figure 4 confirms that the average number of boutons in the
C layers and MIN from the area 18 axons greatly exceeds the number from area 17.

\section{Comparison of the innervation pattern with eye dominance}

We demonstrated previously a marked bias in the feedback from area 17 in that cells driven by the contralateral eye were shown to project preferentially to layer A and those driven by the ipsilateral eye to layer A1 (Murphy and Sillito, 1996). It was again our intention to label area 18 axons arising from the monocular regions at the center of ocular dominance columns to compare their eye preferences and innervation patterns. However, it proved far more difficult to find substantial regions of purely monocular driving in area 18 . This was especially so in the depths of layer VI in which eye preference was seen to switch within very short distances. Thus, the ocular dominance of the area 18 axons is less certain than in the previous experiments, and the results are somewhat less clear.

Of the seventeen axons, we identified eight as having been driven preferentially by the contralateral and nine by the ipsilateral eye. In general, the ipsilateral eye axons are well biased toward the "appropriate" layer A1, with up to 11.6 times as many boutons in this layer compared with the "inappropriate" layer A. For example, the one illustrated in Figure 6 (right) gives off only a few sparse collaterals in layer A before forming a massive arborization, with 3.4 times as many boutons, in layer A1. The axon in Figure 6 (left) likewise has a more extensive arborization and 2.5 times as many boutons in layer $\mathrm{A} 1$ as in layer $\mathrm{A}$. The one in Figure 5, although somewhat unusual, is noteworthy in that it virtually bypasses layer A altogether. Some of the axons that were stained by injecting a contralateral eye column are equally well biased toward layer A. For example, the one illustrated in Figure 2 (left) has a main arborization that is almost entirely restricted to layer A, with only a few collaterals penetrating through the depth of layer A1 to form a second arborization in layer $\mathrm{Cm}$. This axon has 4.3 times as many boutons in the appropriate compared with the inappropriate layer. However, a number of axons that were apparently driven by the contralateral eye are either relatively unbiased, such as the one illustrated in Figure 2 (right) or, in two cases only, are actually biased toward layer A1. Thus, the ipsilateral eye axons are, if anything, more biased than those from area 17, whereas the contralateral eye axons are less so. The distribution of terminals in the A laminae for axons of different eye preference is given in Figure 7. For the area 18 population as a whole, they have on average $65 \%$ of their layer A and A1 terminals in the appropriate layer ( $75 \%$ for ipsilateral eye-driven and $54 \%$ for contralateral eye-driven axons) compared with $35 \%$ in the inappropriate layer. This is a smaller overall bias than that seen for area 17.

One interpretation is that the termination of area 18 axons is disproportionately weighted toward layer A1. A similar situation has been reported for the afferent pathway (Höllander and Vanegas, 1977; LeVay and Ferster, 1977; Geisert, 1985) in which the number of area 18-projecting cells is greater in layer A1 than in layer $\mathrm{A}$. The ratio of contralateral and ipsilateral eye input to area 18 is balanced by a substantial additional projection from magnocellular layer $\mathrm{C}$. The feedback axons were therefore reanalyzed to compare the input to layer A1 with the combined inputs to layers $\mathrm{A}$ and $\mathrm{Cm}$. This maneuver has little effect on the area 17 data but substantially shifts the ratio for area 18. As expected, the contralateral eye axons appear more selective when both contralateral laminae are included in the analysis. For example, 


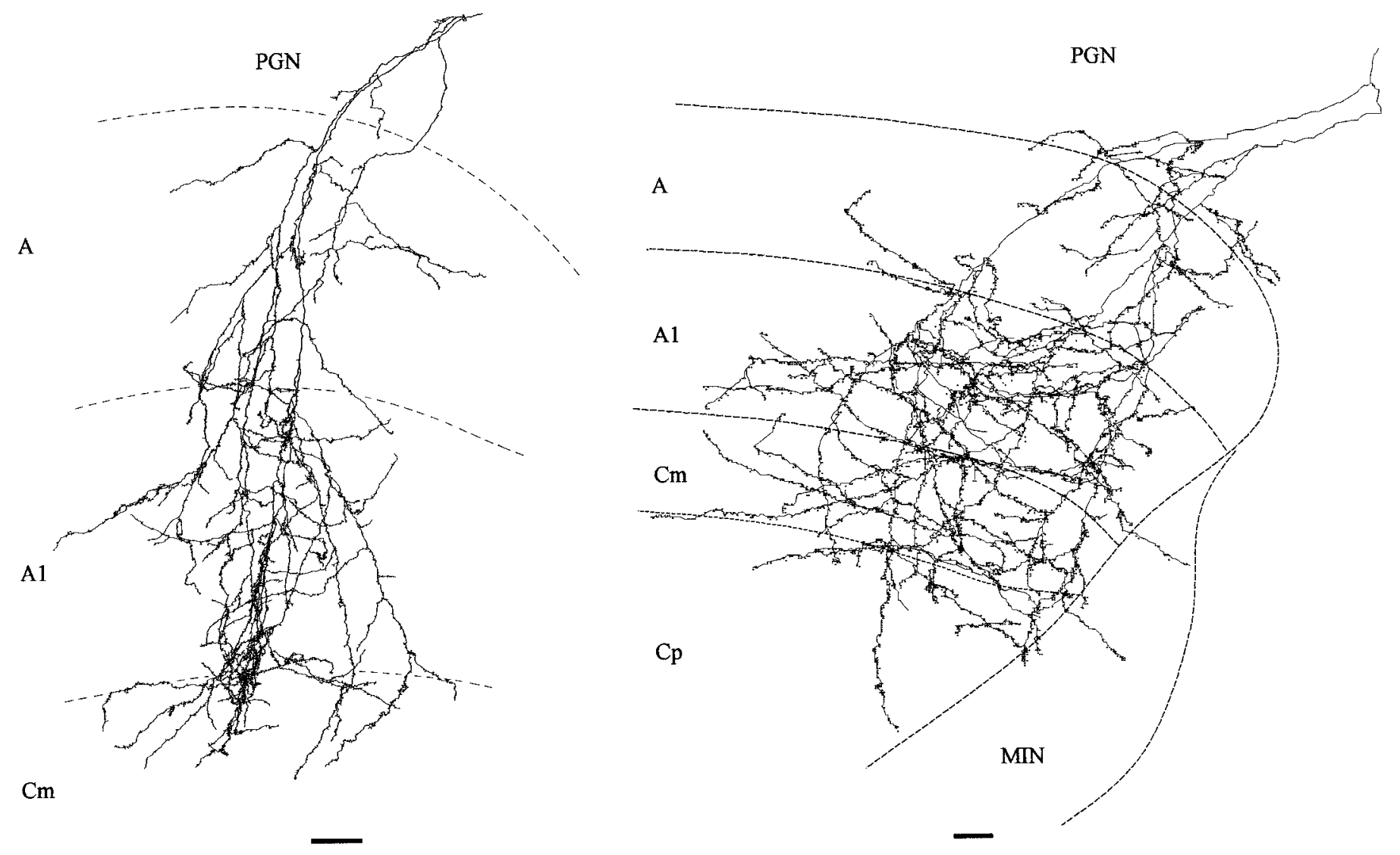

Figure 6. Reconstructions of two corticofugal axons that were stained by injection into the center of ipsilateral eye ocular dominance columns. The first (left) is atypical in that it has no collaterals at all within MIN. Note however that both axons form an extensive arborization within the ipsilateral eye-dominated layer A1 but provide only a sparse input to the contralateral eye-dominated layer A. Surprisingly, both also provide substantial input to layer $\mathrm{Cm}$. Scale bars, $100 \mu \mathrm{m}$. $C p$, Parvocellular C layers.

whereas the axon in Figure 2 (right) has only 1.5 times more boutons in layer $\mathrm{A}$ than layer A1, this ratio increases to 2.2 times when layers A plus $\mathrm{Cm}$ are combined. However, a surprising and potentially important finding is that layer $\mathrm{Cm}$ receives an equally heavy input from axons driven by the ipsilateral eye (Figs. 5, 6). Hence ipsilateral eye axons, and indeed the data for the population as a whole, appear less selective when analyzed in this way.

\section{Comparison of the sublaminar distribution of boutons}

Although the geniculate layers are generally assumed to be homogeneous, there is some evidence that projections both to (Geisert, 1985) and from (Updyke, 1975) area 18 are differentially distributed with respect to the depth of the A layers. These layers were therefore subdivided into top, middle, and bottom thirds, so that the frequency of boutons could be examined separately for each division. The data for area 17 were reanalyzed in the same way for the sake of comparison. Note that five area 18 axons that were drawn using the computer-linked reconstruction system had to be excluded from this section, because it was not possible to apply an equivalent method of analysis.

Both groups of feedback axons were found to have a differential distribution within the body of the A layers (Fig. 8). We analyzed the total bouton count and spread (defined as the total number of sampling windows containing boutons) per sublayer for each of our sampled of axons. For area 18 axons, there is a tendency for both measures to peak in the bottom two-thirds of their preferred layer, giving rise to a small but statistically significant difference

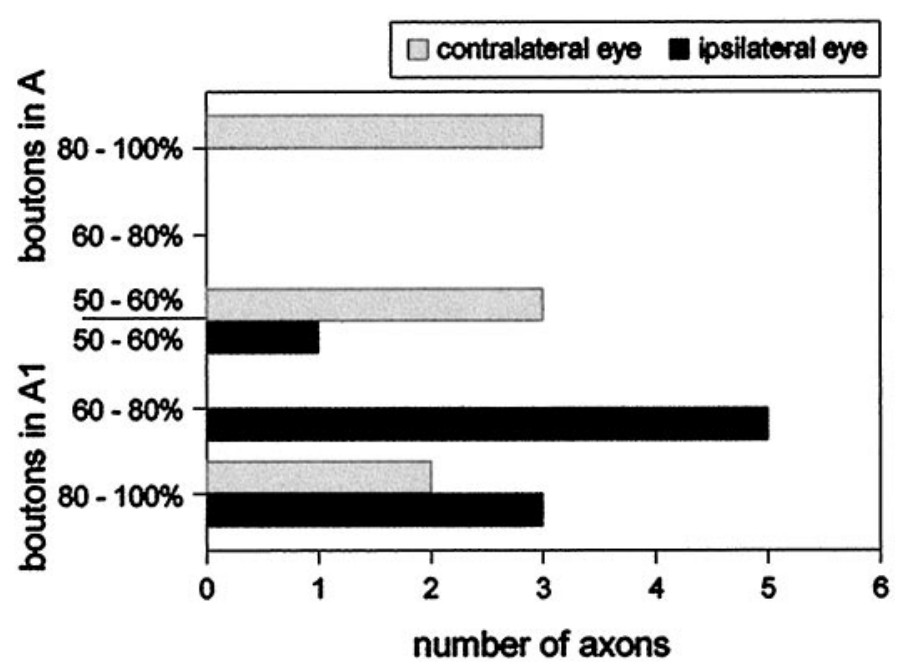

Figure 7. The distribution in the A laminae of area 18 corticofugal boutons, for each of 17 fully reconstructed axons. The data are divided into five categories relating to the proportion of boutons in layer $\mathrm{A}$ as opposed to A1 and are color-coded according to whether the cells of origin responded best to the contralateral ( gray bars) or ipsilateral (black bars) eye. Note that $80-100 \%$ of the boutons in layer A corresponds to $10-20 \%$ in layer A1, and vice versa. Although every axon innervates both layers, those driven by the ipsilateral eye show a marked preference for the ipsilaterally driven layer A1. A reciprocal relationship is not seen for the contralaterally driven axons. 

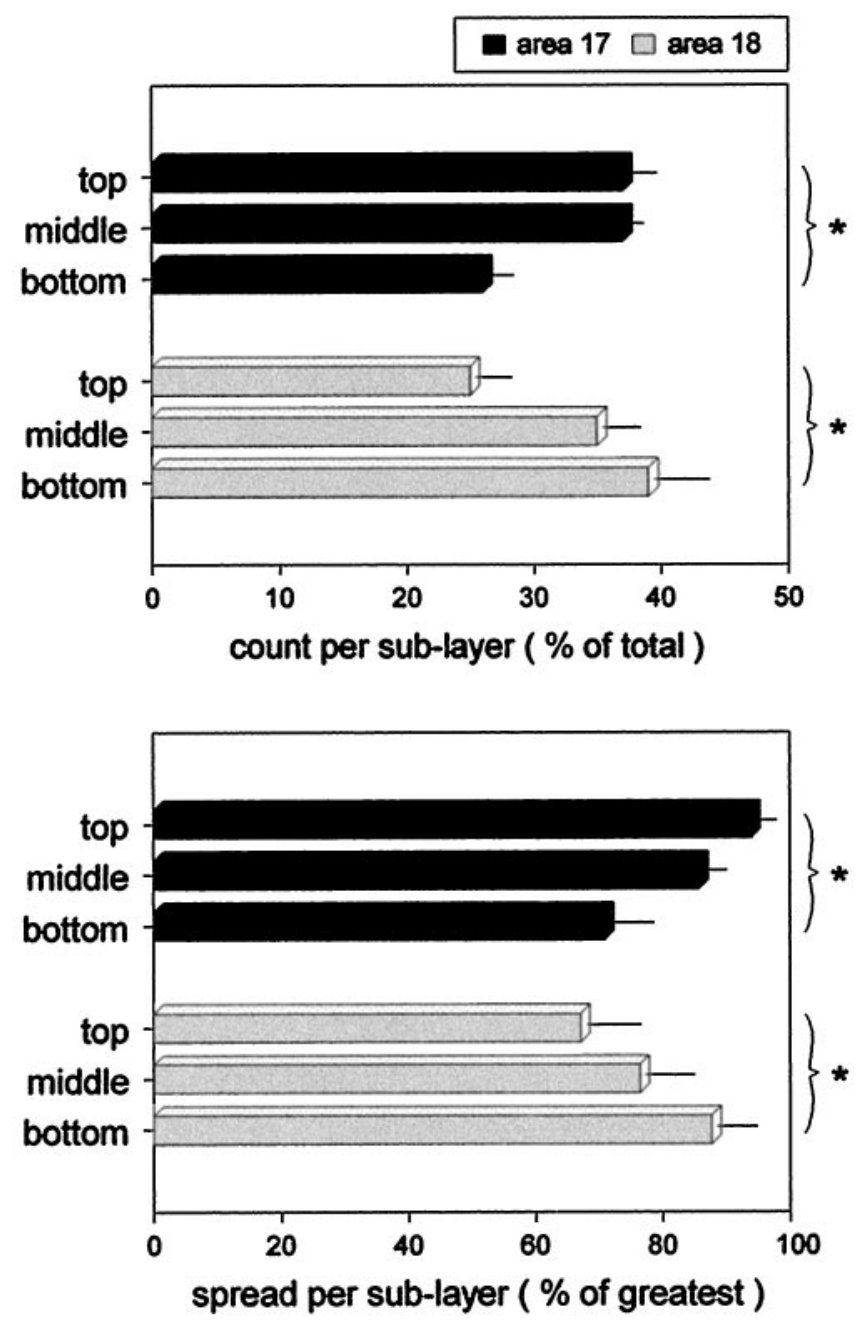

Figure 8. The distribution of boutons from areas 17 and 18 axons, within the top, middle, and bottom third of the preferred A layer of each axon (i.e., the layer receiving the strongest innervation, and hence, in all but 2 cases, the layer matching the eye preference of the axon). Two measures are given: the number of boutons per sublayer (top), expressed as a percentage of the total number for the entire layer, and the mediolateral spread of the arborization in each sublayer (bottom), expressed as a percentage of the greatest value. The difference between the top and bottom compartments is significant in each case (Student's $t$ test; $p<$ $0.05)$. Error bars are $1 \mathrm{SEM} ; n=14$ for area $17 ; n=12$ for area 18 .

between the innervation of the top and bottom compartments of that layer. Thus, contralateral eye axons from area 18 tend to terminate preferentially and arborize more extensively toward the bottom of layer A (Fig. 2), and ipsilateral eye axons arborize more extensively toward the bottom of layer A1 (Figs. 5, 6). The distribution of boutons from area 17 axons shows a reciprocal pattern, with bouton numbers and spread tending to peak within the top two-thirds of the preferred layer, as a result of which there is again a statistically significant difference between the innervation of the top and bottom sublayers. It has been suggested that the area 18 pathway selectively targets the interlaminar zones (Updyke, 1975), not only terminating more densely but extending further laterally within these specific regions. None of our stained axons show this pattern. Long, horizontally directed collaterals are, however, frequently seen in the regions close to the laminar boundaries. This can be seen most clearly in the examples illustrated in Figures 2 and 5.

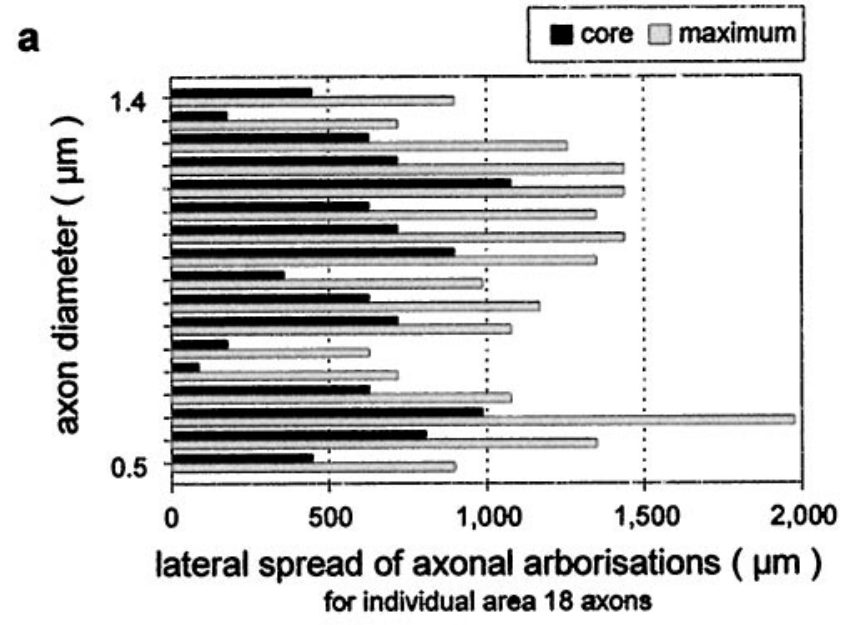

b
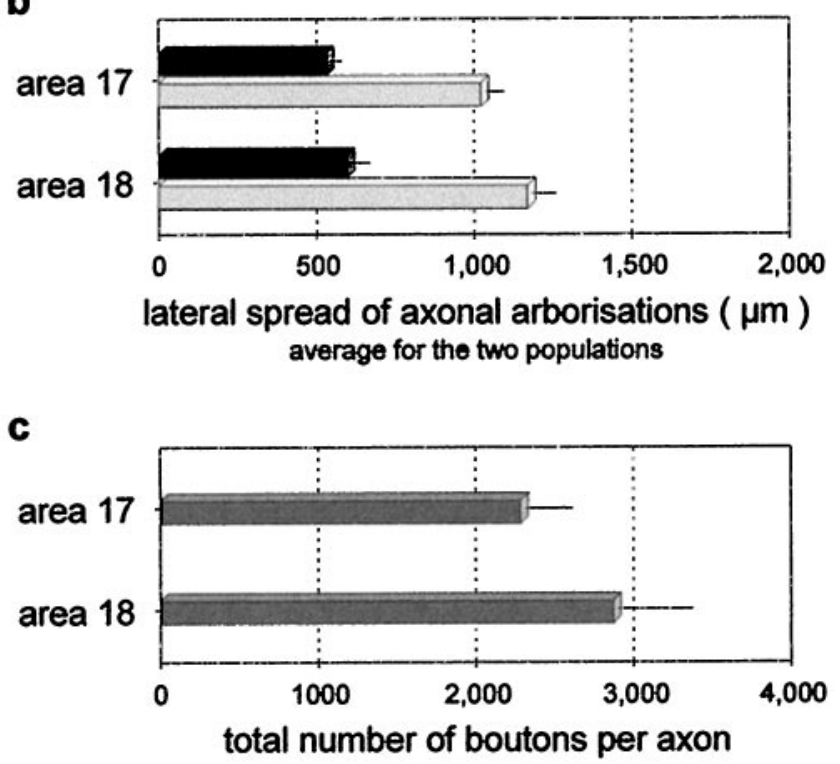

Figure 9. Graphs showing the mediolateral spread of the arborization for each reconstructed area 18 axon $(a)$ and a comparison of the average spread $(b)$ and average number of boutons $(c)$ for the areas 17 and 18 axon populations. In $a$, the data are ranked according to axon diameter, with the thickest at the top and the thinnest at the bottom. The "core" (black bars) is the central region within which the bouton number per $90-\mu \mathrm{m}$-wide strip exceeds half of the maximum value; "maximum" ( gray bars) refers to the greatest tip-to-tip spread of the longest ranging collaterals. Error bars are $1 \mathrm{SEM} ; n=14$ for area $17 ; n=17$ for area 18 .

\section{Comparison of the lateral spread of the corticofugal input}

The area 18 axons show considerable variety as to the shape, pattern, and lateral extent of their intrageniculate arborizations. In these respects, the areas 17 and 18 axons are quantitatively very similar. Figure 9 illustrates several points of comparison. The top part of the figure shows the medial to lateral spread for each area 18 axon individually (Fig. 9a), as well as a histogram comparing the results for areas 17 and 18 (Fig. 9b). As before (Murphy and Sillito, 1996), two measures are given, one showing the size of the central core of input (now defined quantitatively as the region over which the bouton counts per mediolateral compartment exceed half of the maximum value) and the other showing the greatest tip-to-tip distance between the farthest 
reaching branches. Measurements are confined to the A layers, because these are the main target for both pathways. It is clear that, although there is a great deal of variability within each population as a whole, the termination zones of the two pathways are well matched in size (core region: area 17, range of 360-810 $\mu \mathrm{m}$, mean $\pm \mathrm{SEM}$ of $535 \pm 45 \mu \mathrm{m}$; area 18 , range of $180-1080$ $\mu \mathrm{m}$, mean $\pm \mathrm{SEM}$ of $600 \pm 70 \mu \mathrm{m}$; maximum spread: area 17 , range of 540-1350 $\mu \mathrm{m}$, mean $\pm \mathrm{SEM}$ of $1020 \pm 75 \mu \mathrm{m}$; area 18 , range of $630-1980 \mu \mathrm{m}$, mean \pm SEM of $1170 \pm 85 \mu \mathrm{m})$. Likewise (Fig. 9c), there is no significant difference in the average number of boutons per axon (area 17, mean \pm SEM of $2290 \pm 295$; area 18 , mean \pm SEM of $2865 \pm 480)$. Thus, the arborizations are equally widespread and equally sparse.

\section{DISCUSSION}

We have labeled and reconstructed the entire thalamic arborizations of axons in the feedback pathway from area 18 of the visual cortex to the dLGN and compared their morphology and projection patterns with those from area 17 . The results show that there are substantial differences in the laminar distribution of their boutons, which appear to reciprocate the differences seen in the afferent pathways to these cortical areas. However, the results also show a marked similarity in the distribution of their boutons with respect to the geniculate retinotopic map, which is entirely surprising in light of the very different retinotopic organizations of the two areas.

\section{Laminar distribution of the corticogeniculate boutons}

Comparison of the patterns of connectivity of the areas 17 and 18 axons provides several lines of evidence in favor of reciprocity in the corticofugal pathways. For example, the most conspicuous difference between the two pathways is that the area 18 axons provide substantially more input to the C layers and MIN. They therefore target the layers containing the $\mathrm{Y}$ - and W-cells that project to area 18 (Höllander and Vanegas, 1977; LeVay and Ferster, 1977; Geisert, 1980). In contrast, the successfully stained area 17 axons, which terminate primarily within the A layers, appear to reciprocate the distribution of the X- and Y-type geniculate cells from which they receive their own principal input (Murphy and Sillito, 1996).

Detailed quantitative analysis has revealed other differences, which appear to obey the same general rule. Because the axons were stained by injecting into the center of ocular dominance columns, it is reasonable to suppose that their responses were dominated by afferents driven by one or the other eye. Both populations show a bias in their feedback, such that the majority of the axons preferentially innervate the A layer relaying input from that eye. Furthermore, the area 18, but not the area 17, feedback pathway sends a stronger projection to layer A1 compared with layer $\mathrm{A}$, whereas layer $\mathrm{Cm}$ appears to receive equally strong feedback from both ipsilateral and contralateral eye ocular dominance columns. Both of these characteristics recapitulate the patterns seen in the afferent pathways (Höllander and Vanegas, 1977; LeVay and Ferster, 1977; Geisert, 1980, 1985; Boyd and Matsubara, 1996). Finally, there is evidence that the Y-cell pathway to area 18 relays more heavily via the lower portion of each A layer (Bowling and Michael, 1984; Geisert, 1985), whereas the $\mathrm{X}$-cell pathway to area 17 shows the opposite distribution. The boutons from the areas 17 and 18 feedback axons follow a similar pattern, suggesting that they replicate even these most subtle biases. One obvious inference is that the pathways are strictly reciprocal, with each cortical area contacting the specific relay cells that provide afferent input to that area.

Although strict reciprocity would be compatible with the present data and with evidence that areas 17 and 18 boutons synapse with different dendritic profiles in the dLGN (Vidnyánszky and Hámori, 1994), it seems highly unlikely given the substantial cross-talk between the two pathways at other levels. For example, there is evidence that area 18 feedback axons contact inhibitory interneurons belonging to the X-cell pathway (Vidnyánszky and Hámori, 1994), although X-type relay cells project only to area 17 . In addition, areas 17 and 18 receive branching projections from single geniculate $\mathrm{Y}$ cells, so those cells at least would be expected to receive feedback from both areas. Thus, each cortical area must have the potential to influence the relay of retinal information to the other. In this context, it is important to note that area 18 receives input from the thickest, fastest axons in the afferent pathway (Tretter et al., 1975; Höllander and Vanegas, 1977; LeVay and Ferster, 1977; Geisert, 1980) and that the thickest and presumably fastest feedback axons originate in area 18. This loop must therefore provide the first corticogeniculate signal in response to any stimulus condition. Given the relative conduction times of X and Y axons (Cleland et al., 1971, 1976; Tretter et al., 1975), area 18 could well provide a preemptive influence on the responses of X-type relay cells and through them the function of the striate cortex.

\section{Feedback to magnocellular layer $\mathbf{C}$}

One oddity of the primary visual pathway in cats is the absence of an ipsilateral eye layer to match the contralaterally driven layer $\mathrm{Cm}$, because the result is an apparent imbalance in the representation of the two eyes at the thalamic level. It has been suggested that a homologous population of cells might be subsumed within the neighboring ipsilateral eye laminae (Boyd and Matsubara, 1996); indeed, this is one possible explanation for the overrepresentation of layer A1 in the afferent pathway to area 18. Assuming that these cells would receive the same mixture of feedback that is characteristic to layer $\mathrm{Cm}$, this might also explain the apparent anomalies in the pattern of input from area 18. For example, the eye-specific bias in the area 18 feedback to the $\mathrm{A}$ layers is considerably less clear than that for area 17. This could be because of the methodological constraints described earlier or a consequence of too small a sample. However, comparison of the data for contralateral and ipsilateral eye axons suggests that both populations project more strongly than expected to layer A1. Taking note of the fact that layer $\mathrm{Cm}$ receives equal feedback relating to either eye, it is possible that the additional input to layer A1 relates to the presence of Y cells that are homologous to those of layer $\mathrm{Cm}$.

The unique mixture of corticof ugal feedback to layer $\mathrm{Cm}$ could help to explain the different visual response properties of Y cells in the A and C layers (Frascella and Lehmkuhle, 1984; Lee et al., 1992). It also raises possibilities for further study. For example, the $\mathrm{Y}$ cells in layer $\mathrm{Cm}$ are predominantly monocular. However, as with other geniculate cells, they can be influenced by stimulation of their nondominant eye (Suzuki and Kato, 1966; Singer, 1970; Sanderson et al., 1971; Rodieck and Dreher, 1979; Kato et al., 1981; Xue et al., 1987; Guido et al., 1989). Nondominant eye responses can be modified by corticofugal feedback (Schmeilau and Singer, 1977; Varela and Singer, 1987; Murphy and Sillito, 1989) and, because they receive a noneye-specific cortical input, it seems likely that layer $\mathrm{Cm}$ cells should be especially susceptible to this influence. Given that layer $\mathrm{Cm}$ afferents terminate in ipsilat- 
erally as well as contralaterally driven ocular dominance columns (Boyd and Matsubara, 1996) and that layer Cm responds differently to abnormal binocular competition during development (Kato et al., 1981; Garraghty et al., 1989; Spear et al., 1989), the possibility that this layer plays a special role in binocular image processing is worth addressing.

\section{Pattern of connectivity within retinotopic space}

The area 18 axons, like those from area 17 (Murphy and Sillito, 1996), form sparse but extensive arborizations that encompass large portions of the geniculate map of visual space. This suggests that axons from both areas contact cells that respond to stimuli well outside their own classical receptive fields. It might be argued that the spread of the dLGN cell dendrites compensates for the spread of the corticofugal arborizations, leaving little or no actual retinotopic mismatch between the cells of origin and their targets. However, this would work only for the largest geniculate cells and the axons with the smaller arbors. Furthermore, it would only work if the axons specifically avoided making contact with the many cells that partially overlap their arborizations. Otherwise, the spread of the geniculate cell dendrites would increase rather than decrease the spatial divergence in the pathway. In the absence of any evidence to support the alternative, the present results reinforce our belief that the corticof ugal system is involved in global, and not just local, information processing.

The fact that there is no significant difference in the mediolateral extent of the areas 17 and 18 arborizations is extremely surprising. Receptive fields in area 18 are substantially larger than those in area 17 (Tretter et al., 1975; Orban and Kennedy, 1981), and the retinotopic map in area 18 is substantially more compressed along the mediolateral axis (Tusa et al., 1978, 1979; Cynader et al., 1987). The existing evidence therefore suggests that, although the axons match anatomically, there are substantial differences in the functional connectivity of the two pathways. It is possible that the explanation lies in our relatively small sample size. However, it is our impression that the difference in areas 17 and 18 receptive field sizes is far less in layer VI than in the superficial layers. If so, then the spatial organization of the feedback pathways may be matched from both physiological and anatomical points of view. Given the substantial differences that exist between the two areas as a whole, this is likely to be functionally important. Therefore, we are currently in the process of analyzing the relationship between the three-dimensional spread of the feedback connections and quantitatively derived receptive field maps for a population of areas 17 and 18 layer VI cells to determine whether this impression is correct.

\section{REFERENCES}

Bowling DB, Michael CR (1984) Terminal patterns of single, physiologically characterized optic tract fibers in the cat's lateral geniculate nucleus. J Neurosci 4:198-216.

Boyapati J, Henry GH (1984) Corticofugal axons in the lateral geniculate nucleus of the cat. Exp Brain Res 53:335-340.

Boyd JD, Matsubara JA (1996) Laminar and columnar patterns of geniculocortical projections in the cat: relationship to cytochrome oxidase. J Comp Neurol 365:659-682.

Cleland BG, Dubin MW, Levick WR (1971) Sustained and transient neurones in the cat's retina and lateral geniculate nucleus. J Physiol (Lond) 217:473-496.

Cleland BG, Levick WR, Morstyn R, Wagner HG (1976) Lateral geniculate relay of slowly conducting retinal afferents to cat visual cortex. J Physiol (Lond) 255:299-320.

Cudeiro J, Sillito AM (1996) Spatial frequency tuning of orientation- discontinuity-sensitive corticof ugal feedback to the cat lateral geniculate nucleus. J Physiol (Lond) 490:481-492.

Cynader MS, Swindale NV, Matsubara JA (1987) Functional topography in cat area 18. J Neurosci 7:1401-1413.

Dreher B, Leventhal AG, Hale PT (1980) Geniculate input to cat visual cortex: a comparison of area 19 with areas 17 and 18 . J Neurophysiol 44:804-826.

Erisir A, Van Horn SC, Sherman SM (1997) Relative numbers of cortical and brainstem inputs to the lateral geniculate nucleus. Proc Natl Acad Sci USA 94:1517-1520.

Erisir A, Van Horn SC, Sherman SM (1998) Distribution of synapses in the lateral geniculate nucleus of the cat: differences between laminae A and $\mathrm{A} 1$ and between relay cells and interneurons. J Comp Neurol 390:247-255.

Frascella J, Lehmkuhle S (1984) A comparison between Y-cells in A-laminae and lamina $\mathrm{C}$ of cat dorsal lateral geniculate nucleus. J Neurophysiol 52:911-920.

Garraghty PE, Roe AW, Chino YM, Sur M (1989) Effects of convergent strabismus on the development of physiologically identified retinogeniculate axons in cats. J Comp Neurol 289:202-212.

Geisert EE (1980) Cortical projections of the lateral geniculate nucleus in the cat. J Comp Neurol 190:793-812.

Geisert EE (1985) The projection of the lateral geniculate nucleus to area 18. J Comp Neurol 238:101-106.

Geisert EE, Langsetmo A, Spear PD (1981) Influence of the corticogeniculate pathway on response properties of cat lateral geniculate nucleus. Brain Res 208:409-415.

Guido W, Tumosa N, Spear PD (1989) Binocular interactions in the cat's dorsal lateral geniculate nucleus. I. Spatial-frequency analysis of the responses of $\mathrm{X}, \mathrm{Y}$, and $\mathrm{W}$ cells to nondominant-eye stimulation. J Neurophysiol 62:526-543.

Guillery RW (1966) A study of Golgi impregnations from the dorsal lateral geniculate nucleus of the adult cat. J Comp Neurol 128:21-50.

Höllander H, Vanegas H (1977) The projections from the lateral geniculate nucleus onto the visual cortex in the cat. J Comp Neurol 173:519-536.

Hubel DH, Wiesel TN (1965) Receptive fields and functional architecture in two nonstriate visual areas (18 and 19) of the cat. J Neurophysiol 28:229-289.

Kalil RE, Chase R (1970) Corticofugal influence on activity of lateral geniculate neurons in the cat. J Neurophysiol 33:459-474.

Kato H, Bishop PO, Orban GA (1981) Binocular interaction on monocularly discharged lateral geniculate and striate neurons in the cat. J Neurophysiol 46:932-951.

Kawamura S, Sprague JM, Niimi K (1974) Corticofugal projections from the visual cortex to the thalamus, pretectum, and superior colliculus in the cat. J Comp Neurol 158:339-362.

Lee D, Lee C, Malpeli JG (1992) Acuity-sensitivity trade-offs of X and Y cells in the cat lateral geniculate complex: role of the medial interlaminar nucleus in scotopic vision. J Neurophysiol 68:1235-1247.

LeVay S, Ferster D (1977) Relay cell classes in the lateral geniculate nucleus of the cat and the effects of visual deprivation. J Comp Neurol 172:563-584.

Marrocco RT, McClurkin JW, Young RA (1982) Modulation of lateral geniculate nucleus cell responsiveness by visual activation of the corticogeniculate pathway. J Neurosci 2:256-263.

McCart RJ, Henry GH (1994) Visual corticogeniculate projections in the cat. Brain Res 653:351-356.

Montero VM (1991) A quantitative study of synaptic contacts on interneurons and relay cells of the cat lateral geniculate nucleus. Exp Brain Res 86:257-270.

Movshon JA, Thompson ID, Tolhurst DJ (1978) Spatial and temporal contrast sensitivity of neurones in areas 17 and 18 of the cat's visual cortex. J Physiol (Lond) 283:101-120.

Murphy PC, Sillito AM (1987) Corticofugal feedback influences the generation of length tuning in the visual pathway. Nature 329:727-729.

Murphy PC, Sillito AM (1989) The binocular input to cells in the feline dorsal lateral geniculate nucleus (dLGN). J Physiol (Lond) 415:393-408.

Murphy PC, Sillito AM (1996) Functional morphology of the feedback pathway from area 17 of the cat visual cortex to the lateral geniculate nucleus. J Neurosci 16:1180-1192.

Murphy PC, Grieve KL, Sillito AM (1993) Effects of vasoactive intestinal polypeptide on the response properties of cells in area 17 of the cat visual cortex. J Neurophysiol 69:1465-1474. 
Orban GA, Callens M (1977) Receptive field types of area 18 neurones in the cat. Exp Brain Res 30:107-123.

Orban GA, Kennedy H (1981) The influence of eccentricity on receptive field types and orientation selectivity in areas 17 and 18 of the cat. Brain Res 208:203-208.

Orban GA, Kennedy H, Maes H (1981a) Response to movement of neurons in area 17 and 18 of the cat: velocity sensitivity. J Neurophysiol 45:1043-1058.

Orban GA, Kennedy H, Maes H (1981b) Response to movement of neurons in areas 17 and 18 of the cat: direction selectivity. J Neurophysiol 45:1059-1073.

Price DJ, Ferrer JMR, Blakemore C, Kato N (1994) Functional organization of corticocortical projections from area 17 to area 18 in the cat's visual cortex. J Neurosci 14:2732-2746.

Richard D, Gioanni Y, Kitsikis A, Buser P (1975) A study of geniculate unit activity during cryogenic blockade of primary cortex in the cat. Exp Brain Res 22:235-242.

Robson JA (1983) The morphology of corticofugal axons to the dorsal lateral geniculate nucleus in the cat. J Comp Neurol 216:89-103.

Robson JA (1984) Reconstruction of corticogeniculate axons in the cat. J Comp Neurol 225:193-200.

Rodieck RW, Dreher B (1979) Visual suppression from nondominant eye in the lateral geniculate nucleus: a comparison of cat and monkey. Exp Brain Res 35:465-477.

Sanderson KJ, Bishop PO, Darian-Smith I (1971) The properties of binocular receptive fields of lateral geniculate neurons. Exp Brain Res 13:178-207.

Schmeilau F, Singer W (1977) The role of visual cortex for binocular interactions in the cat lateral geniculate nucleus. Brain Res 120:354-361.

Sillito AM, Cudeiro J, Murphy PC (1993) Orientation sensitive elements in the corticofugal influence on centre-surround interactions in the dorsal lateral geniculate nucleus. Exp Brain Res 93:6-16.
Sillito AM, Jones HE, Gerstein GL, West DC (1994) Feature-linked synchronization of thalamic relay cell firing induced by feedback from the visual cortex. Nature 369:479-482.

Singer W (1970) Inhibitory binocular interaction in the lateral geniculate body of the cat. Brain Res 18:165-170.

Spear PD, McCall MA, Tumosa N (1989) W-and Y-cells in the Clayers of the cat's lateral geniculate nucleus: normal properties and effects of monocular deprivation. J Neurophysiol 61:58-73.

Suzuki H, Kato E (1966) Binocular interaction at cat's lateral geniculate body. J Neurophysiol 29:909-920.

Tretter F, Cynader M, Singer W (1975) Cat parastriate cortex: a primary or secondary visual area. J Neurophysiol 38:1099-1113.

Tusa RJ, Palmer LA, Rosenquist AC (1978) The retinotopic organization of area 17 (striate cortex) in the cat. J Comp Neurol 177:213-236.

Tusa RJ, Rosenquist AC, Palmer LA (1979) Retinotopic organization of areas 18 and 19 in the cat. J Comp Neurol 185:657-678.

Updyke BV (1975) The patterns of projection of cortical areas 17, 18, and 19 onto the laminae of the dorsal lateral geniculate nucleus in the cat. J Comp Neurol 163:377-396.

Varela FJ, Singer W (1987) Neuronal dynamics in the visual corticothalamic pathway revealed through binocular rivalry. Exp Brain Res 66:10-20.

Vidnyánszky Z, Hámori J (1994) Quantitative electron microscopic analysis of synaptic input from cortical areas 17 and 18 to the dorsal lateral geniculate nucleus in cats. J Comp Neurol 349:259-268.

Weber AJ, Kalil RE (1987) Development of corticogeniculate synapses in the cat. J Comp Neurol 264:171-192.

Wilson JR, Friedlander MJ, Sherman SM (1984) Fine structural morphology of identified X- and Y-cells in the cat's lateral geniculate nucleus. Proc R Soc Lond B Biol Sci 221:411-436.

Xue J-T, Ramoa AS, Carney T, Freeman RD (1987) Binocular interaction in the dorsal lateral geniculate nucleus of the cat. Exp Brain Res 68:305-310. 